\title{
Primary liver cells cultured on carbon nanotube substrates for liver tissue engineering and drug discovery applications
}

\begin{abstract}
Here, we explore the use of two- and three-dimensional scaffolds of multiwalled-carbon nanotubes (MWNTs) for hepatocyte cell culture. Our objective is to study the use of these scaffolds in liver tissue engineering and drug discovery. In our experiments, primary rat hepatocytes, the parenchymal (main functional) cell type in the liver, were cultured on aligned nanogrooved MWNT sheets, MWNT yarns, or standard 2-dimensional culture conditions as a control. We find comparable cell viability between all three culture conditions but enhanced production of the hepatocyte-specific marker albumin for cells cultured on MWNTs. The basal activity of two clinically relevant cytochrome P450 enzymes, CYP1A2 and CYP3A4, are similar on all substrates, but we find enhanced induction of CYP1A2 for cells on the MWNT sheets. Our data thus supports the use of these substrates for applications including tissue engineering and enhancing liver-specific functions, as well as in in vitro model systems with enhanced predictive capability in drug discovery and development.
\end{abstract}

Keyword: Carbon nanotubes; Tissue engineering; Liver toxicity testing; Rat hepatocytes; Albumin; CYP enzyme activities 